Original Article

\title{
Principles of diagnostics and therapy in homeopathy, information approach.
}

\author{
Tatiana V. Akaeva ${ }^{1 *}$, Karen N. Mkhitaryan ${ }^{1}$, Mikhail Yu. Gotovskiy $^{1^{* *}}$ \\ 1 Limited Liability Company Center of Intellectual Medical Systems "IMEDIS" Moscow, Russia. E-mail: * $\underline{\text { akaeva_tatyana@mail.ru, }}$ **mchitaryan@mail.ru
}

\begin{abstract}
Background: Homeopathic tradition comes from the empiric vision "simila similium", developed by Samuel Hahnemann. But experience of the homeopathy's development, acquired during two and a half century, its show this principle is insufficient for diagnosis and therapy homeopathic and informational preparations. Within the limits of this principle for example is impossible: a) to explain the action of organic preparations, "electronic copies" of usual drugs, potentiated RNA and DNA, revealed in various tissues, organs and systems, and many other information preparations; b) to justify and describe more widely, from the point of view of the contemporary science, the physiological mechanisms of the action of homeopathic preparations, especially those that have in high and ultra-high dilutions, in particular, "electronic" potencies. c) to create informational preparations with predetermined pathogenesis-and programmed action, inclusively homeopathic preparations.

Aims: The authors are proposing to be considered the homeopathic preparations high and ultrahigh dilutions and, also, the informational preparations as control signals (CS), which are transmitting in the organism the information about supplementary condition, of which the organism it is necessary to continue its vital activity. The information about the presence of a similar condition causes in the organism the rfaction of ataptation that the authors propose to be called reaction of advance adaptation.
\end{abstract}

Methodology: The simple shaping of some possible reactions of advance adaptation leads to the examination of two of these types of reaction: reaction of resistance and reaction of observance. The reaction of resistance explains and allows detailing the principle "simila similium" advanced by S. Hahnemann. The reaction of observance explains the effectiveness of organic preparations and allows to be made informational preparations (control signals (CS) in principle of another type of action than the homeopathic one. The result of the presented principles, authors and their colleagues received a series of information preparations, including various gerontoprotectors and regenerative amplifiers, especially: Triton Regeneration, Triton Metamorphosis, Proliferation of Neuroblasts, Proliferation of Fibroblasts, Syvstar, and others. Conducted evaluation of the effectiveness of therapy new classes of homeopathic and information remedy, including gerontoprotectors and amplifiers of regeneration. For diagnostics and therapy, and fabrication necessary preparations and marker $\mathrm{CMH}$ was used the integrated system for electropuncture diagnostic «IMEDIS-EXPERT». The preparations were indicated based on the principle of observance and resistance, according to the diagnostic test through VRT-BRT method (Vegetative Resonance Test - Bio Resonance Therapy), within the framework of the system-nosological 
approach developed by the authors, and account the individual complex chrono-semantic marker $(\mathrm{CMH})$, taken from the patient.

Results: Clinical testing showed high efficiency of new homeopathic and information remedy. The clinical experiments of 1075 patients have demonstrated a high effectiveness of these preparations.

Conclusion: New class preparations used within the system-nosological approach gives of $86 \%$ a positive result. The results obtained are not feasible with the aid of usual homeopathy.

Keywords: homeopathic preparation, informational preparation, control signal (CS), complex chrono-semantic marker (CMH).

Received: March 1, 2018. Accepted: April 26, 2018.

(C) International Journal of High Dilution Research.

Not for commercial purposes. 